\section{Suicidio y cirugía bariátrica: un estudio de la evidencia}

\author{
ARTURO ROIZBLATT ${ }^{1}$, DANIEL ROIZBLATT ${ }^{2}$, \\ FRANCISCA SOTO-AGUILAR B. ${ }^{3}$
}

\section{Suicide risk after bariatric surgery}

Bariatric surgery is considered the most effective treatment for severe obesity and its benefits include improvement in medical comorbidities. However, a higher rate of suicides after this type of surgery has been reported. We performed a literature review on the subject, and concluded that the mentioned increase in suicide rates, compared to the general population, is probably caused by conditions that the patient had before surgery, especially psychiatric disorders such as depression or eating disorders. These are risk factors for suicide, and are more common in the population with indication for bariatric surgery. Therefore, it is necessary to thoroughly evaluate these patients before surgery searching for suicide risk factors, deriving them to a mental health professional if necessary and follow their mental health after surgery. Considering that the literature on the topic is inconsistent, further research is needed.

(Rev Med Chile 2016; 144: 1171-1176)

Key words: Bariatric Surgery; Depression; Obesity; Suicide.

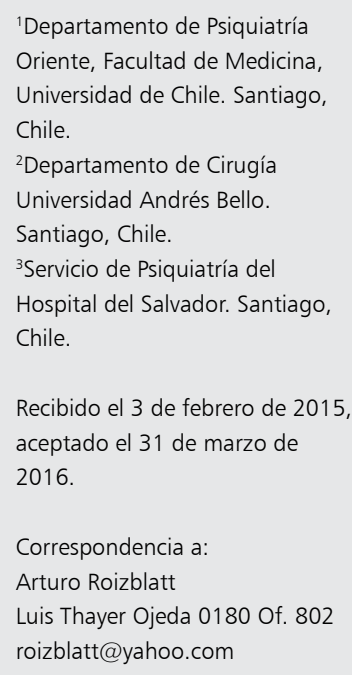

\section{L} a cirugía bariátrica, considerada actualmente el tratamiento más efectivo para la obesidad severa ${ }^{1-3}$, incluye entre sus beneficios, además de la disminución en el peso, una mejoría en comorbilidades médicas como diabetes o hipertensión, y también en la calidad de vida ${ }^{4}$. Sin embargo, aunque son escasos los estudios longitudinales sobre la materia con seguimiento a largo plazo, en algunos sí se ha observado una asociación entre cirugía bariátrica y ciertas patologías, sobre todo trastornos psiquiátricos y suicidio ${ }^{2,5,6}$.

Entre los factores descritos en la alta incidencia de suicidios en el grupo en referencia, están la obesidad en sí misma, alteraciones en la absorción de alcohol o medicamentos poscirugía bariátrica, el no logro en la baja de peso esperada o el aumento del peso perdido poscirugía, tabaquismo, abuso de alcohol y el Índice de Masa Corporal (IMC), además de factores genéticos ${ }^{5,7,10}$.

Por otra parte, diversas condiciones se han visto asociadas por sí mismas con el suicidio, particularmente la depresión, el trastorno de personalidad limítrofe y el trastorno de estrés postraumático ${ }^{11-13}$. Desde esta perspectiva, entre los candidatos a cirugía bariátrica es frecuente la comorbilidad psiquiátrica, con prevalencias de hasta $66 \%{ }^{14-16}$. De hecho, se ha observado que aproximadamente $10 \%$ de los individuos obesos severos que buscan cirugía bariátrica reportan una historia de intentos suicidas previos, siendo esta una prevalencia mayor que la de la población general ${ }^{17}$. Es así como es difícil evaluar si la relación entre la cirugía bariátrica y el suicidio es causal, o más bien una asociación estadística.

El objetivo de este estudio es examinar, a partir de la evidencia, la posibilidad de una relación entre la cirugía bariátrica y el suicidio, y evaluar qué factores pueden ser de riesgo en el desarrollo de esta patología.

\section{Método}

Se realizó una revisión bibliográfica de la literatura, incluidos 48 artículos publicados entre 
los años 1997 y 2015, utilizando los términos "cirugía bariátrica", "obesidad" y "suicido" para la búsqueda en Pubmed.

\section{Asociación entre suicidio y cirugía bariátrica}

\section{Tasa de suicidios en población sometida a cirugía bariátrica}

Los hallazgos de diversos estudios han demostrado un aumento en la tasa de suicidios en los pacientes sometidos a cirugía bariátrica. Adams et al. ${ }^{18}$ realizaron un estudio de cohorte retrospectivo para evaluar las tasas de mortalidad en un plazo de 18 años, por causas relacionadas con la obesidad (enfermedad cardiovascular, diabetes y cáncer) y por otras. Con tal fin, compararon un grupo de pacientes que se sometieron a cirugía bariátrica con un grupo control de pacientes con obesidad mórbida a los que no se les realizó esta cirugía. Los resultados se parearon según género, edad e IMC (esta última variable se midió en todos los pacientes en la fecha correspondiente al período precirugía del primer grupo, independientemente del IMC que presentaban al momento del fallecimiento). Entre los resultados de este estudio, destaca que en el grupo de pacientes que se sometieron a cirugía bariátrica, las tasas de mortalidad fueron considerablemente más bajas en las siguientes categorías: $92 \%$ menos por diabetes $(\mathrm{p}=0,005), 59 \%$ menos por cardiopatía coronaria $(\mathrm{p}=0,006)$ y $60 \%$ menos por cáncer $(\mathrm{p}=0,001)$. Sin embargo, al analizar las muertes no causadas por estas enfermedades (incluido suicidio, accidentes relacionados con drogas, envenenamiento sin intención suicida y otras muertes), se constata que en el grupo sometido a cirugía bariátrica hubo 1,58 veces más muertes en comparación con el grupo control $(p=0,04)$.

En Estados Unidos de Norteamérica, al analizar Tindle et al. ${ }^{3}$ la tasa de suicidios en pacientes sometidos a esta cirugía, encontraron una cifra de $6,6 / 10.000$, que incluía 5,2 por 10.000 mujeres y 13,7 por 10.000 hombres. Ambos valores aparecieron como considerablemente más altos que los de la población general, que en Estados Unidos de Norteamérica corresponden a una tasa de suicidios de $0,7 / 10.000$ en mujeres y 2,4/10.000 en hombres $^{19}$. Por su parte, Omalu et al. ${ }^{6}$ realizaron una investigación para evaluar las causas de muerte en pacientes sometidos a cirugía bariátrica, también encontrando en ellos una mayor tasa de suicidios. Además, Peterhänsel et al. ${ }^{20}$ llevaron a cabo una revisión sistemática de la evidencia existente sobre la tasa de suicidios en pacientes sometidos a esta cirugía, en la que incluyeron el estudio de Tindle et al. $^{3}$. La tasa de suicidios estimada en este caso fue de 4,1/10.000.

\section{Factor temporal entre la cirugía bariátrica y trastornos psiquiátricos}

Existen hallazgos interesantes en cuanto a la temporalidad de los cambios en la salud mental de los pacientes sometidos a cirugía bariátrica. Los investigadores Assimakopoulos et al. ${ }^{21}$ estudiaron los efectos de esta cirugía en cuanto a ansiedad, síntomas depresivos, IMC y función sexual, obteniendo resultados de mejoría significativa al primer año poscirugía. Sin embargo, como se describe a continuación, el seguimiento de estos pacientes en plazos menores a un año es insuficiente en la evaluación de dichas patologías.

Junaid et al. ${ }^{16}$ en Canadá, realizaron una evaluación acerca de conductas autolesivas en 8.815 pacientes sometidos a cirugía bariátrica antes y después de la misma, observando que la incidencia de emergencias por autolesiones aumentó en 50\% en el período posoperatorio, pero la mayoría tuvo lugar en el segundo y tercer año tras la operación. De estos pacientes que presentaron conductas autolesivas, casi la totalidad correspondió a aquellos que ya tenían una historia de trastornos psiquiátricos.

En el estudio ya mencionado de Tindle et al. ${ }^{3}$, se observó que $30 \%$ de los suicidios se presentaba en los dos primeros años poscirugía y 70\% en los tres primeros años. Lo anterior pudiera explicarse por el deterioro en los síntomas depresivos después del primer año de cirugía, como lo demostraron Mitchell et al. ${ }^{22}$, quienes compararon la presencia de sintomatología depresiva antes y después de esta cirugía. Según sus hallazgos, $28,3 \%$ de los pacientes incluidos en su estudio reportó sintomatología depresiva precirugía, valor que descendió a $8,4 \%$ al final del primer año de la cirugía bariátrica, para aumentar posteriormente a $12,2 \%$ y a $15,6 \%$ al segundo y tercer año, respectivamente. Así, si bien los casos de sintomatología depresiva son menos que antes de la cirugía $(\mathrm{p}<0,001)$, van en aumento con el paso del tiempo $(\mathrm{p}<0,01)$. 


\section{Asociación entre obesidad y trastornos psiquiátricos}

\section{Obesidad y trastorno depresivo}

Es conocida la relación entre trastornos de malnutrición, como la obesidad y el bajo peso, con la depresión y otros trastornos psiquiátricos. En el caso de pacientes obesos con depresión, según un estudio realizado por Gao et al. ${ }^{23}$, los síntomas depresivos mejorarían cuando el paciente disminuye su peso. No obstante, los mismos autores hacen referencia a lo complejo de aislar el factor peso en estos pacientes, por la gran cantidad de comorbilidades y otras enfermedades mentales asociadas que pueden influir en tal sintomatología.

\section{Obesidad y trastornos alimentarios}

En cuanto a los trastornos alimentarios que se presentan en los pacientes obesos, muchos de ellos se deben a conductas de alimentación que se ven influidas por las emociones ${ }^{24}$, por lo que, independientemente del tratamiento médico que se realice para la obesidad, la prevención en cuanto a la reincidencia en la malnutrición requiere de un tratamiento psiquiátrico simultáneo. Además, se ha observado que los pacientes que presentan este tipo de trastornos, en cualquiera de sus grados, antes de someterse a la cirugía bariátrica, tendrán más ansiedad y depresión que los pacientes sin estos trastornos ${ }^{25,26}$.

\section{Obesidad y suicidio}

Los estudios sobre la relación entre obesidad y suicidio han desembocado en diversos hallazgos. En algunos se ha observado una relación positiva entre IMC y riesgo suicida, sugiriendo que a mayor IMC habría mayor riesgo de suicidio ${ }^{2,6,14,17,26,27}$; en otros, se ha establecido una asociación negativa, según la cual a mayor IMC habría menor riesgo de suicidio $^{28-30}$. Por último, también existen estudios en los que no se ha observado ninguna relación entre ambos factores ${ }^{31-33}$. Cabe destacar que, al menos en el sexo femenino, se ha observado que si bien la asociación entre obesidad y suicidio puede ser inversamente proporcional, la ideación suicida y los intentos suicidas aumentan a mayor IMC ${ }^{32,34}$. Por otra parte, en población adolescente se ha observado que la obesidad tiene una asociación con la ideación suicida, aunque no con intentos suicidas propiamente tales ${ }^{34}$.

Una cierta evidencia interesante sobre la rela- ción entre IMC y suicidio surge cuando se evalúan por separado los diferentes grados de obesidad: se ha observado que pacientes con obesidad grado III (IMC $\geq 40$ ) presentan una prevalencia de conductas suicidas de hasta $33 \%$ en el caso de las mujeres y de $13 \%$ en hombres, y específicamente de intentos suicidas en $27 \%$ de las mujeres y $13 \%$ en los hombres. Curiosamente, para obesidad grados I y II no se ha observado la misma relación; más bien, se ha visto una prevalencia menor para conductas suicidas en mujeres con obesidad grado II al compararlas con la del grado ${ }^{15}$.

La relación entre IMC, depresión y conducta suicida está aún en continuo debate, con estudios contradictorios ${ }^{15} \mathrm{y}$ resultados muchas veces poco claros. Tales discordancias podrían explicarse por varias razones: en primer lugar, en algunos estudios que han separado los grupos de diferentes valores de IMC y su relación con suicidios, se ha observado que la asociación, si bien es positiva, no es lineal: es cuadrática. De esta manera, si se compara una persona de peso normal y otra obesa, quizás no se encuentre una diferencia significativa entre el riesgo de suicidio en una y en otra; sin embargo, si se compara una persona de peso normal con otra que presenta un IMC extremadamente alto, sí existe diferencia, con mayor riesgo para la última $^{14}$. Por otro lado, existen también algunas variables que influyen en el mayor o menor riesgo suicida de una persona obesa u obesa mórbida; así, los pacientes que tienen una percepción correcta de su peso tienen un aumento significativo en su ideación suicida ${ }^{28,34}$, al igual que aquellos que sienten la percepción de sus seres queridos respecto de su condición de obesidad ${ }^{14}$.

\section{Discusión}

La evidencia existente muestra claramente una tendencia hacia un aumento en la tasa de suicidios en los pacientes sometidos a cirugía bariátrica en comparación con la población general. Sin embargo, de acuerdo con lo expuesto, no se ha logrado aún establecer una relación causal, debido a las muchas otras variables intervinientes. Sí se puede observar que la preexistencia de ciertos factores en los pacientes aumenta el riesgo de evolucionar en esta patología; entre ellos, la obesidad severa, el consumo problemático de alcohol, el tabaquismo, la depresión, el trastorno de personalidad 
limítrofe, trastornos alimentarios y el trastorno de estrés postraumático. Ello es más válido aún si estos pacientes ya tienen una historia de intentos suicidas previos.

Se ha observado al respecto que en la población sometida a cirugía bariátrica, a diferencia de otro tipo de intervenciones quirúrgicas, ya hay riesgos elevados de suicidio, tanto antes como después de la misma ${ }^{35}$. Se puede concluir, entonces, que se trata de pacientes que deben considerarse como grupo de riesgo, y que es necesario actuar en consonancia con tal diagnóstico. En tal perspectiva, a continuación, se plantean algunas sugerencias respecto de posibles medidas a considerar en estos casos.

\section{Evaluación preoperatoria}

Al evaluar a un paciente para someterlo a cirugía bariátrica, es importante explorar su historia psiquiátrica y, específicamente, pesquisar la existencia de trastornos afectivos e indicadores de riesgo suicida, tanto de quien se va a operar como de sus familiares. Invariablemente, el paciente debe ser derivado para evaluación especializada que haga sugerencias prequirúrgicas o manifieste si hay aspectos que contraindiquen la intervención. El cirujano debe ser acucioso en la revisión de la evaluación realizada por el equipo de salud mental, de tal manera que no sea considerada un "mero trámite" precirugía. También es necesario tener presente que muchas veces los pacientes pueden intentar falsear la información entregada, sabiendo que eventualmente se les puede rechazar como candidatos a la cirugía, de no cumplir con los requisitos previamente establecidos. De no tenerse en consideración los aspectos señalados, puede llegarse a subestimar los factores psicológicos asociados al suicido en tales pacientes ${ }^{15,35}$.

Otro elemento en el análisis preoperatorio es el uso de métodos de detección precoz, como lo es el MMPI-2-RF (Minnesota Multiphasic Personality Inventory), creado por Tarescavage et al. $^{36}$, que conjuntamente con observaciones conductuales e información clínica permiten complementar la exploración.

\section{Evaluación posoperatoria y seguimiento}

Si a pesar de presentar antecedentes de riesgo, el paciente tiene indicación de cirugía, es importante considerar algún tipo de seguimiento posoperatorio. Seguimientos con psiquiatras y educación desarrollada en torno a la alimentación, con sistemas conductuales-motivacionales, han demostrado ser más eficientes en la disminución de síntomas depresivos que sistemas de evaluación posoperatoria no estructurados ni específicos en tratar este tipo de afecciones ${ }^{1}$.

Es importante destacar que los estudios centrados en evaluar la relación entre la cirugía bariátrica y los síntomas depresivos, debieran considerar un seguimiento de los pacientes mayor a un año poscirugía, ante la posibilidad, comprobada en algunos casos, de encontrarse mejoras de tales síntomas durante el primer año poscirugía que sólo tienen carácter momentáneo.

\section{Conclusiones}

Si bien la evidencia muestra una mayor incidencia de suicidio en pacientes sometidos a cirugía bariátrica en comparación con la población general, para establecer una asociación causal es necesario analizar de forma independiente, diferentes variables que suelen estar presentes simultáneamente en estos pacientes.

Considerando que la cirugía bariátrica es cada vez más frecuente, se hace indispensable tener en consideración el tema expuesto -su asociación con el suicidio o con sintomatologías depresivas- en la evaluación previa a la intervención, así como en el seguimiento posterior a corto y largo plazo.

Es también importante, en estudios futuros, acotar cuáles son las psicopatologías asociadas directamente a la cirugía bariátrica; $y$, al mismo tiempo, establecer la magnitud que puede alcanzar para el paciente obeso y su riesgo suicida el saber los riesgos que trae consigo la obesidad, la aislación social que puede producir, e incluso los potenciales peligros de ser sometido a una cirugía no menor ${ }^{35}$.

Creemos, con este trabajo, entregar herramientas a los equipos de salud que trabajan con este tipo de pacientes, que les faciliten la identificación y tratamiento adecuado de aquellos que tienen indicación de intervenciones psiquiátricas pre y poscirugía bariátrica.

\section{Referencias}

1. Petasne M, Campa A, Samiri S, Sosa J. Comprehensive behavioral-motivational nutrition education improves depressive symptoms following bariatric surgery: A ran- 
domized, controlled trial of obese Hispanic Americans. J Nutr Educ Behav 2013; 45 (6): 620-6.

2. Diniz Mde F, Moura LD, Kelles SM, Diniz MT. Longterm mortality of patients submitted to Roux-en-Y gastric bypass in Public Health System: High prevalence of alcoholic cirrhosis and suicides. Arq Bras Cir Dig 2013; 26 (1): 53-6.

3. Tindle HA, Omalu B, Courcoulas A, Marcus M, Hammers J, Kuller LH. Risk of suicide after long-term follow-up from bariatric surgery. Am J Med 2010; 123: 1036-42.

4. Elder KA, Wolfe BM. Bariatric surgery: A review of procedures and outcomes. Gastroenterology 2007; 132: 2253-71.

5. Mitchell JE, Crosby R, De Zwaan M, Engel S, Roerig J, Steffen K, et al. Possible risk factors for increased suicide following bariatric surgery. Obesity (Silver Spring) 2013; 21 (4): 665-72.

6. Omalu BI, Ives DG, Buhari AM, Lindner JL. Death rates and causes of death after bariatric surgery for Pennsylvania residents, 1995 to 2004. Arch Surg 2007; 142 (10): 923-8.

7. Schneider B, Lukaschek K, Baumert J, Meisinger C, Erazo N, Ladwig KH. Living alone, obesity, and smoking increase risk for suicide independently of depressive mood findings from the population-based MONICA/ KORA Augsburg cohort study. J Affect Disord 2014; 152-154: 416-21.

8. Dahl JK, Eriksen L, Vedul-Kjelsås E, Strommen M, Kulseng B, Marvik R, et al. Depression, anxiety, and neuroticism in obese patients waiting for bariatric surgery: Differences between patients with and without eating disorders and subthreshold binge eating disorders. Obes Res Clin Pract 2014; 6 (2): 139-47.

9. Hsu LK, Benotti, PN, Dwyer J, Roberts SB, Saltzman E, Shikora S, et al. Nonsurgical factors that influence the outcome of bariatric surgery: A review. Psychosom Med 1998; 60: 338-46.

10. Chojnicka I, Fudalej S, Walczak A, Wasilewska K, Fudalej $\mathrm{M}$, Stawinski P, et al. Inverse association between obesity predisposing FTO genotype and completed suicide. PLoS ONE 2014: 9 (9). Disponible en: http:// www.plosone.org/ [Consultado el 1 de agosto de 2015].

11. Cavanagh JT, Carson AJ, Sharpe M, Lawrie SM. Psychological autopsy studies of suicide: A systematic review. Psychol Med 2003; 33 (5): 947.

12. Gradus JL, Qin P, Lincoln AK, Miller M, Lawler E, Lash TL. The association between adjustment disorder diagnosed at psychiatric treatment facilities and completed suicide. Clin Epidemiol 2010; 2: 23-8.

13. Harris EC, Barraclough B. Suicide as an outcome for mental disorders. A meta-analysis. Br J Psychiatry 1997; 170: 205-28.

14. Dutton GR, Bodell LP, Smith AR, Joiner TE. Examination of the relationship between obesity and suicidal ideation. Int J Obes (Lond) 2013; 37 (9): 1282-6.

15. Wagner B, Klinitzke G, Brähler E, Kersting A. Extreme obesity is associated with suicidal behavior and suicide attempts in adults: Results of a population-based representative sample. Depress Anxiety 2013; 30: 975-81.

16. Bhatti JA, Nathens AB, Thiruchelvam D, Grantcharov T, Goldstein BI, Redelmeier DA. Self-harm emergencies after bariatric surgery: A population-based cohort study. JAMA Surg 2015: oct 7: 1-7. Disponible en: http://archsurg.jamanetwork.com/article.aspx?articleid=2448916 [Consultado el 31 de octubre de 2015].

17. Lester D, Iliceto P, Pompili M, Girardi P. Depression and suicidality in obese patients. Psychol Rep 2011; 108 (2): 367-8.

18. Adams TD, Gress RE, Smith SC, Halverson C, Simper SC, Rosamond WD, et al. Long term mortality after gastric bypass surgery. N Engl J Med 2007; 357: 753-61.

19. Health, United States: With Chartbook on Trends in the Health of Americans [Internet]. Washington: US Department of Health and Human Services. 2005. Table 46, Death rates for suicide, according to sex, race, Hispanic origin and age: United States, selected years 1950-2002; [page 238]. Available from: http://www.cdc. gov/nchs/data/hus/hus05.pdf.

20. Peterhänsel C, Petroff D, Klinitzke G, Kersting A, Wagner B. Risk of completed suicide after bariatric surgery: A systematic review. Obes Rev 2013; 14 (5): 369-82.

21. Assimakopoulos K, Karaivazoglou K, Panayiotopoulos S, Hyphantis T, Iconomou G, Kalfarentzos F. Bariatric surgery is associated with reduced depressive symptoms and better sexual function in obese female patients: A one-year follow-up study. Obes Surg 2011; 21 (3): 3626.

22. Mitchell JE, King WC, Chen J-Y, Devlin MJ, Flum D, García L, et al. Course of depressive symptoms and treatment in the longitudinal assessment of bariatric surgery (LABS-2) study. Obesity (Silver Spring) 2014; 22 (8): 1799-806.

23. Gao S, Juhaeri J, Reshef S, Dai WS. Association between Body Mass Index and suicide, and suicide attempt among British adults: The health improvement network database. Obesity 2013; 21 (3): 334-42.

24. Taube-Schiff M, Van Exan J, Tanaka R, Wnuk S, Hawa $\mathrm{R}$, Sockalingam S. Attachment style and emotional eating in bariatric surgery candidates: The mediating role of difficulties in emotion regulation. Eat Behav 2015; 18: 36-40. 
25. Chen EY, Fettich KC, McCloskey MS. Correlates of suicidal ideation and/or behavior in bariatric-surgery-seeking individuals with severe obesity. Crisis 2012: 33 (3): 137-43.

26. Brewer-Smyth K. Obesity, traumatic brain injury, childhood abuse, and suicide attempts in females at risk. Rehabil Nurs 2014; 39 (4): 183-91.

27. Heneghan HM, Heinberg L, Windover A, Rogula T, Schauer PR. Weighing the evidence for an association between obesity and suicide risk. Surg Obes Relat Dis 2012; 8 (1): 98-107.

28. Kim JS, Lee K. The relationship of weight-related attitudes with suicidal behaviors in Korean adolescents. Obesity (Silver Spring) 2010; 18 (11): 2145-51.

29. Zhang J, Yan F, Li Y, McKeown RE. Body mass index and suicidal behaviors: A critical review of epidemiological evidence. J Affect Disord 2013; 148 (2-3): 147-60.

30. McCarthy JF, Ilgen MA, Austin K, Blow FC, Katz IR. Associations between body mass index and suicide in the veterans affairs health system. Obesity (Silver Spring) 2014; 22 (1): 269-76.

31. Henriksen CA, Mather AA, Mackenzie CS, Bienvenu OJ, Sareen J. Longitudinal associations of obesity with affective disorders and suicidality in the Baltimore epidemiologic catchment area follow-up study. J Nerv Ment Dis 2014; 202 (5): 379-85.

32. Klinitzke G, Steinig J, Blüher M, Kersting A, Wagner B. Obesity and suicide risk in adults--a systematic review. J Affect Disord 2013; 5145 (3): 277-84.

33. Shah A. The relationship between obesity and elderly suicide rates: A cross-national study. J Inj Violence Res 2010; 2 (2): 105-9.

34. Zeller MH, Reiter-Purtill J, Jenkins TM, Ratcliff MB. Adolescent suicidal behavior across the excess weight status spectrum. Obesity (Silver Spring) 2013; 21 (5): 1039-45.

35. Chen EY, Fettich KC, Tierney M, Cummings H, Berona J, Weissman J, et al. Factors associated with suicide ideation in severely obese bariatric surgery-seeking individuals. Suicide Life Threat Behav 2012; 42 (5): 541-9.

36. Tarescavage AM, Windover A, Ben-Porath YS, Boutacoff LI, Marek RJ, Ashton K, et al. Use of the MMPI-2-RF suicidal/death ideation and substance abuse scales in screening bariatric surgery candidates. Psychol Assess 2013; 25 (4): 1384-9. 